\title{
Linguistique et technologie culturelle : L'exemple du métier à tisser vertical berbère
}

cultural technology and linguistics: the exemple of berber vertical loom

\section{Claude Lefébure}

\section{(2) OpenEdition \\ 1 Journals}

Édition électronique

URL : https://journals.openedition.org/tc/4994

DOI : $10.4000 /$ tc. 4994

ISSN : 1952-420X

Éditeur

Éditions de l'EHESS

\section{Édition imprimée}

Date de publication : 30 juin 2010

Pagination : 115-135

ISSN : 0248-6016

\section{Référence électronique}




\section{LINGUISTIQUE ET TECHNOLOGIE CULTURELLE}

\section{L'exemple du métier à tisser vertical berbère}

in Techniques et culture, Bulletin de l'Équipe de recherche 191 (3) 1978: 84-148

Le propos de cette contribution est de montrer, sur l'exemple du métier à tisser vertical utilisé par les femmes berbérophones d'Afrique du Nord, l'intérêt de l'enquête lexicographique et des analyses linguistiques pour les études de technologie culturelle ${ }^{1}$.

Il a déjà été posé qu'entre autres critères susceptibles de justifier le découpage en séquences d'une chaîne opératoire pouvait figurer la référence à un changement de terme vernaculaire (Cresswell 1976: 13). L'importance du discours des acteurs en tant qu'expression d'une partie au moins de leurs connaissances techniques a d'autre part été soulignée (Lemonnier 1976 : 104). Ces deux manières d'aborder l'énonciation indigène ont en commun de n'envisager du discours que ce qu'il peut transmettre dans l'ordre de la dénotation.

On sait cependant que dans toute culture de nombreux signes linguistiques sont investis, en plus de leur sens de base, de sens contextuels ${ }^{2}$. Cette polysémie est source d'associations extra-notionnelles qui, sans altérer le sens dénoté, le colorent, y ajoutent des images subsidiaires, lui confèrent des valeurs expressives $^{3}$. La technologie culturelle ne peut ignorer cet univers des connotations. Une recherche des liaisons articulant le domaine par elle a priori appréhendé comme technique à d'autres sphères de la vie sociale ne peut pas se priver d'être orientée, de l'intérieur de la culture, vers ces liaisons ${ }^{4}$. 


\section{Métiers à tisser d’Afrique du Nord}

Les métiers à tisser en usage en Afrique du Nord illustrent trois des types de la classification d'André Leroi-Gourhan (1971 : 278-296):

1. Métier à un rang de lisses, présent sous les deux formes:

a) du métier vertical, sur cadre,

b) du métier horizontal, sans bâti;

2. Métier à deux rangs de lisses, pédales et poulies;

3. Métier « aux cartons » permettant de tisser des étoffes étroites à chaîne cordée.

Étant donné la situation linguistique ${ }^{5}$ des pays du Maghreb, la localisation des types de métiers n'est pas sans incidence sur le vocabulaire qui s'y rapporte. Ainsi les lexiques des métiers citadins (Bel et Ricard 1913 ; Lapanne-Joinville 1940) sont-ils exclusivement arabes tandis que ceux des métiers ruraux sont soit arabes soit berbères ${ }^{6}$.

Nous n'envisagerons que le métier vertical à un rang de lisses des campagnardes berbérophones. D'une part c'est avec le métier horizontal des mêmes l'un des deux types qu'il m'a été loisible d'observer sur le terrain de mes recherches dans le Sud-Est du Maroc. D'autre part et surtout il s'agit de l'appareil à tisser sur l'exemple duquel la démonstration envisagée m’a paru le mieux susceptible d'aboutir en raison de l'ampleur d'une documentation déjà ordonnée et étudiée (Bynon [- désormais B. -] 1963). [...]

\section{Afrique du Nord: localisation des parlers berbères}

Sont portés sur la carte les seuls toponymes et ethniques cités dans le cours de l'article.

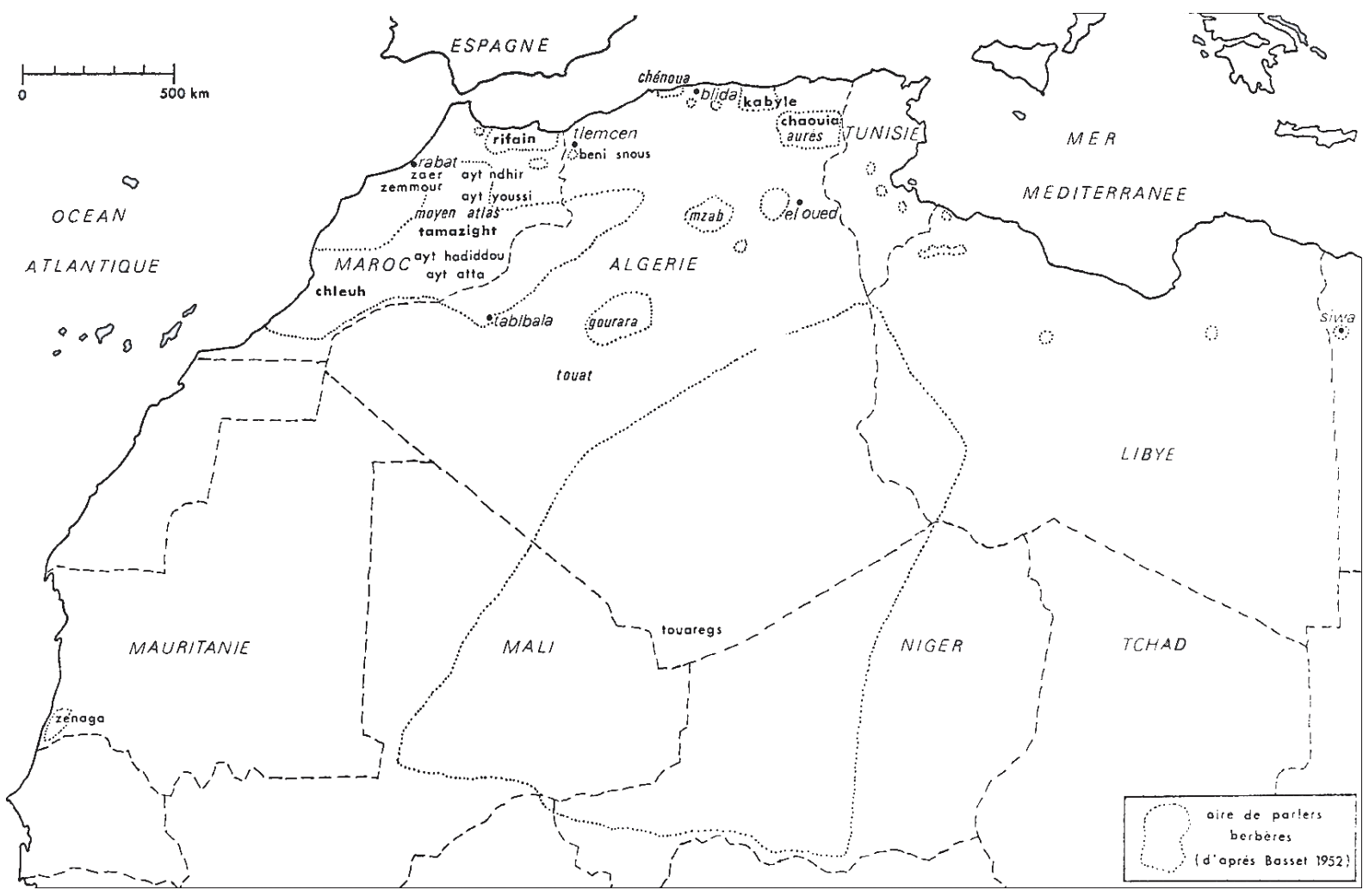




\section{Technologie et vocabulaire du métier vertical berbère}

Planche 1

Principe de fonctionnement du métier vertical à un rang de lisses et chaîne longue (modèle en usage chez les berbérophones du Maroc central)

1. Déroulement de la chaine enroulée sur l'ensouple supérieure.

2. Enroulement du tissage sur l'ensouple inférieure.

A. Barre d'écartement en position haute ouverture du pas. B. Barre d'écartement en position basse ouverture du pas inverse.

c. Variante

avec deux baguettes d'enverjure (ne change rien au mécanisme).

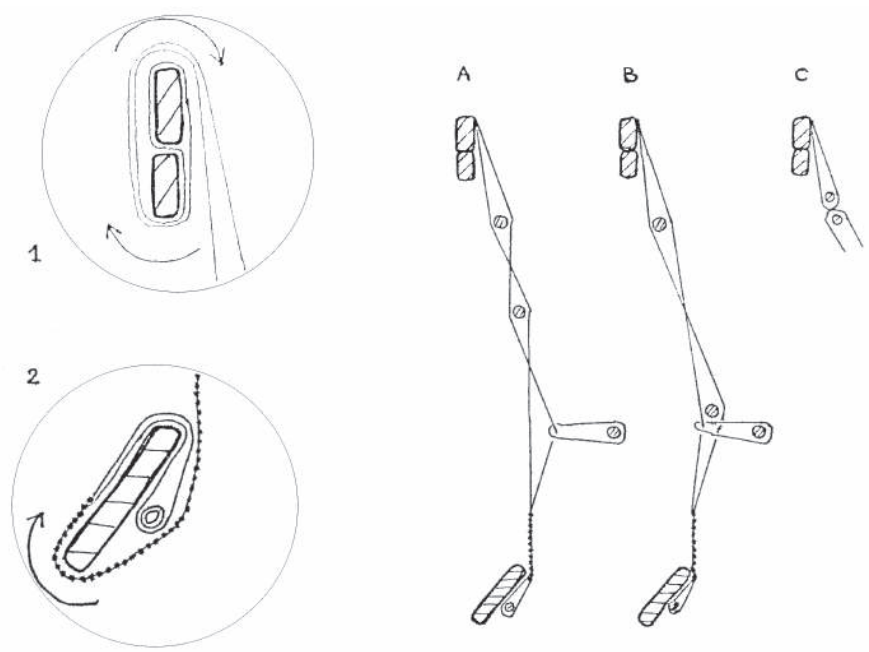

[Appellations]

Avant de détailler les éléments constitutifs de ce métier voyons comment on l'appelle dans son ensemble et quelles remarques sont à faire sur cette appellation (B. 1963 : 33-70). Hormis chez les Zenaga en Mauritanie et chez les Touaregs, populations qui ignorent l'appareil, et à la limite orientale du berbère dans l'oasis de Siwa où on le connaît, tous les parlers soumis à enquête ${ }^{7}$ proposent le même nom, de genre masculin. Transcrivons-le au singulier $a z T a$, pluriel izdwan ou, moins répandu, iztawn. Le mot est à mettre en relation avec le verbe $z d$ « tisser » (mais aussi « tresser, tricoter »).

Quel est le champ sémantique de ce terme? D’abord $a z T a$ ne réfère jamais aux éléments du métier avant leur assemblage pour tendre la chaîne. Ceux-ci sont désignés, quand individuellement par leur nom propre, quand collectivement par une expression signifiant « les instruments de l'azTa », ou « les bois de l'azTa ». Par contre le mot s'entend, avant l'utilisation de ces éléments, dès qu'on commence à parler de l'ourdissage de la chaîne. [...] Enfin, quand commence le tissage proprement dit, deux autres sens de azTa se font jour: " action de tisser et produit de cette action, tissu » ${ }^{8}$. La traduction de $a z T a$ par « métier à tisser » ne s'impose donc en vérité jamais. La situation est différente dans les parlers arabes d'Afrique du Nord où les noms menwel, mensež, seddâya, etc. du métier à tisser vertical à un rang de lisses sont de véritables noms d'instrument.

Examinons maintenant dans le détail la technologie et le lexique du métier berbère.

\section{Les montants (B. 1963 : 101-121)}

Les côtés verticaux du cadre sur lequel est tendue la chaine consistent en deux poteaux de bois plus ou moins bien taillés, les montants. Leurs extrémités inférieures sont simplement posées sur le sol. Pour immobiliser les poteaux, on les attache par le haut entre eux et à des éléments fixes alentour. La soixantaine de notations dont on dispose permet 


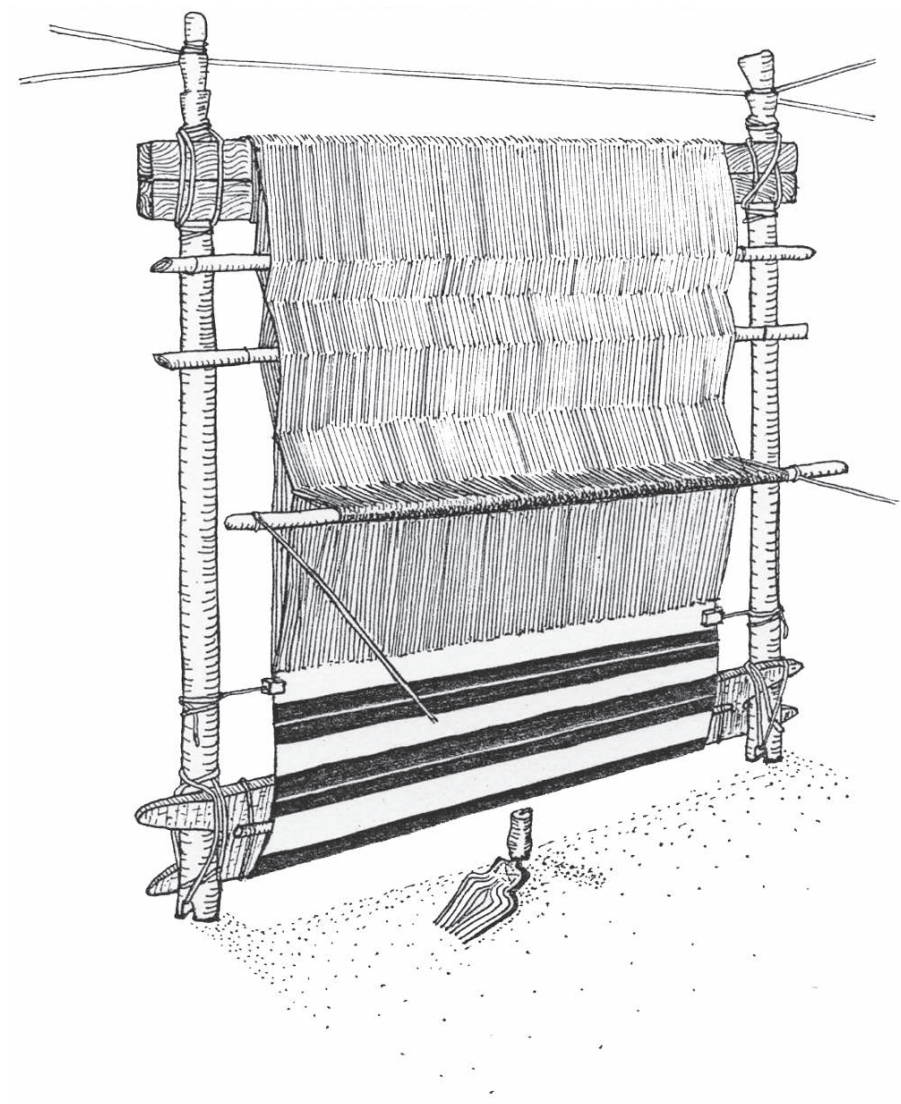

d'identifier quatre noyaux consonantiques, ou racines, différents, autrement dit quatre appellations. Elles se présentent toutes au féminin (soit avec un $t$ initial toujours, et souvent entre deux $t$ ).

La moins fréquente d'entre elles, de racine WQF, n'est attestée qu'en trois points du Rif avec le mot tawQaft, pl. tiwQafin, et à Siwa où l'on a noté waqafa. Elle dérive de l'arabe weqqāf(a), nom d'agent intensif du verbe ūqef: « se dresser, se tenir debout ». [...]

Toujours notée tirslt, pl. tirsal, l'appellation fondée sur la racine RSL est plus répandue que la précédente puisqu'on l'a relevée plusieurs fois au Mzab, où elle paraît la seule connue, chez les Chleuhs, et au moins une fois dans le domaine de la tamazight. Dans ce dernier domaine, une masse de notations indiquent pour le mot, rencontré sous une forme identique ou avec une voyelle $a$ à la place de $i$, le sens de « montant vertical de la tente » et aussi, moins fréquemment, celui de « pilier de soutènement de la toiture d'une maison ». Cette deuxième appellation n'apparaît pas plus spécialisée que la première. Elle en serait l'équivalent berbère. [...]

Une troisième appellation, qui a pour racine RGL, semble dominer ses concurrentes dans le Rif et être exclusive en Kabylie; on l'a relevée aussi au Chenoua. Avec un a dans le Rif taragla (pl. tiragliwin) et un i en Kabylie tiriglit (pl. tirigliwin) les notations intercalent une voyelle entre les deux radicales $R$ et $G$, ce qui confère au mot une structure inhabituelle en berbère. N'ont la même dans cette langue que les mots tarubya: « garance », de rubia, nom latin de cette plante, et tagiawsa: « chose », qui a été rapproché du latin causa (même sens). Plusieurs auteurs font d'ailleurs dériver taragla du latin regula: « règle, et généralement toute barre droite de bois ou de métal ». [...]

\section{Planche 2}

Métier à tisser vertical nord-africain, en usage chez les berbérophones du Maroc central (d'après J. Bynon, in Recherches sur le vocabulaire du tissage en Afrique du Nord, modifié). 
Une dernière appellation fait à elle seule l'objet de trois cinquièmes des notations et se trouve, sauf dans le Mzab et en Kabylie, partout répandue; tamndut ou timndut, pl. timndwin en sont les formes typiques, qui révèlent une racine NDW. Il existe en berbère un verbe de même racine, $n d u$ : « franchir, traverser ».

\section{Les ensouples (B. 1963 : 71-100)}

La chaîne du métier vertical nord-africain est longue. On la tend entre deux fortes traverses horizontales fixées aux montants, l'une au ras du sol, l'autre environ deux mètres au-dessus, les ensouples. L'ensouple supérieure est dite dérouleuse parce qu’au début toute la réserve de fil de chaîne y est enroulée pour être déroulée au fur et à mesure de l'avancement du travail; l'ensouple inférieure, sur laquelle s'enroule le tissu, est nommée ensouple enrouleuse ou ensoupleau. Les ensouples inférieure et supérieure peuvent être plus ou moins différentes mais leur capacité d'enroulement et de déroulement s'équivalent toujours à peu près, de manière à ce qu'en imprimant le même nombre de rotations sur elles-mêmes à chacune de ces traverses le transfert de la chaîne s'effectue sans imposer d'excessives corrections

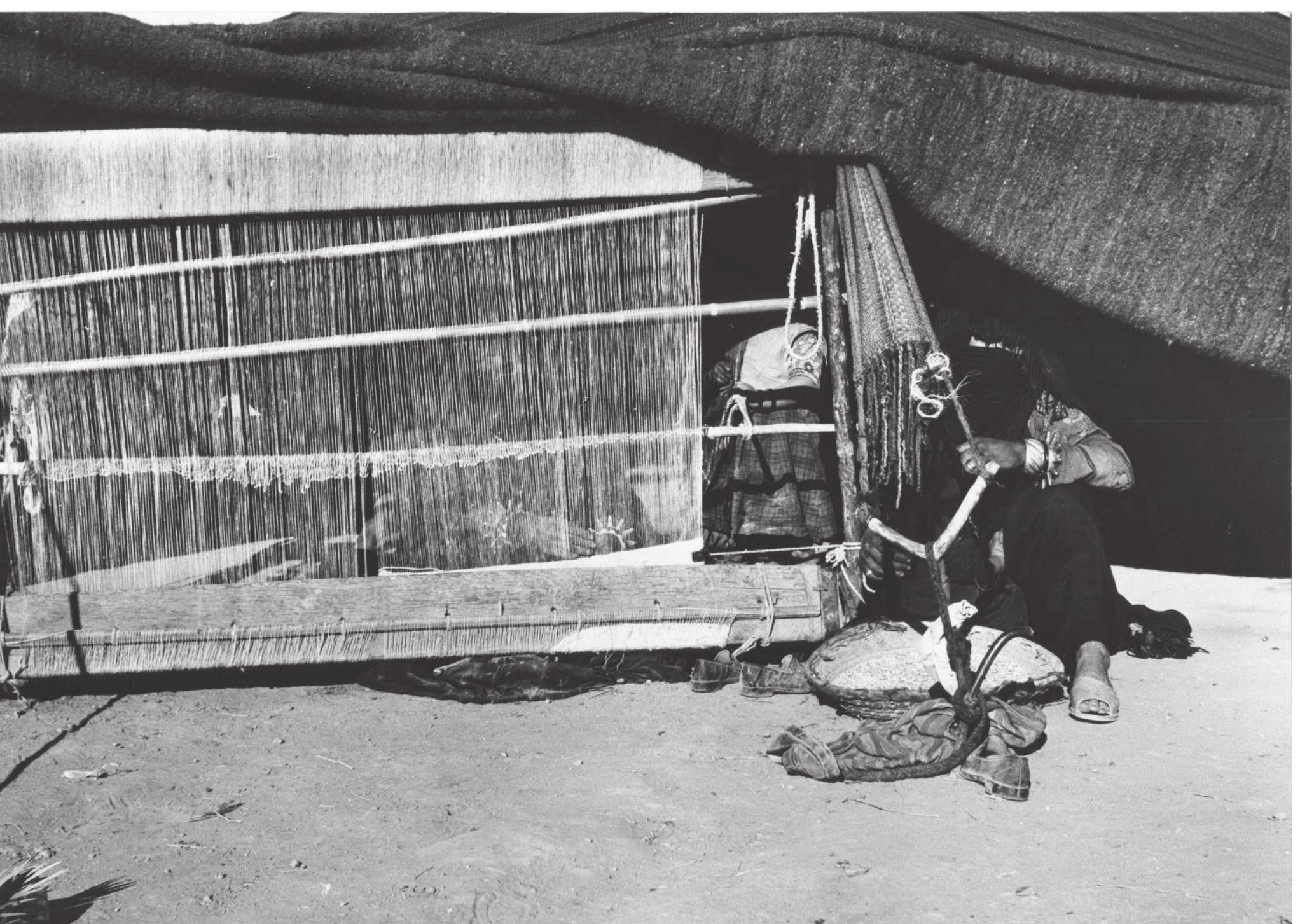


d'écartement pour lui conserver sa tension. Cela reste vrai chez les Imazighen où l'ensouple supérieure est, en fait constituée de deux madriers semblables serrés l'un au-dessus de l'autre lors de l'enroulement de la réserve de chaîne. [...] Sauf chez les Chleuhs qui le percent aux deux bouts d'un trou par où passe le bas des montants, l'ensoupleau présente toujours une fourche à chaque extrémité. Une dizaine d’œillets ménagés le long de son axe médian servent au passage d'une cordelette qui permet de retenir la chaîne.

On retrouve avec la dénomination des ensouples la situation prévalant pour le nom du métier tout entier: un seul terme est utilisé d'un bout à l'autre du domaine. Au pluriel ce terme désigne partout les deux ensouples, au singulier il réfère soit à l'une ou à l'autre d'entre elles indistinctement soit à l'ensoupleau précisément. Ce dernier cas se présente lorsque, en raison de son caractère composite, l'ensouple supérieure reçoit un nom spécial. $g$ pouvant être régionalement réalisé $\check{z}$, la racine de ce terme unique appliqué aux ensouples se présente en fait sous les deux formes FGG et FŽŽ. La première forme est à la base des notations recueillies chez les Chleuhs, les Imazighen et les Kabyles; la seconde à la base de celles effectuées dans le Rif et dans toute l'Algérie moins la Kabylie. Dans ces dernières régions, on entend partout afŽaž, pl. if Žažn, avec un vocalisme que l'on retrouve chez les Kabyles et les Imazighen - afGag, pl. ifGagn - mais non chez les Chleuhs, qui disent ifGig, pl. ifGign. Ni en arabe ni en berbère n'existe de verbe vivant pour témoigner du sens de cette racine.

La dénomination spéciale de l'ensouple dérouleuse composite en usage chez les Imazighen est le pluriel tig̈dwin, le singulier tag்Wda désignant l'une quelconque des deux barres qui la constituent. La racine est restée vivante en chleuh, où l'on connaît le verbe agud: « être rectiligne ». [...]

\section{Les lisses (B. 1963 : 205-216)}

À hauteur des épaules de la tisseuse quand elle est assise à son travail et de son côté par rapport au plan du cadre s'avance le harnais du métier, c'est-à-dire le dispositif qui avec le concours du roseau d'écartement rend possible le croisement alternatif des deux nappes de fils de chaîne. Ce harnais consiste en une barre de lisse, mince mais qui doit être rigide, autour de laquelle est enroulée une cordelette maillant à chacun de ses tours un fil de la nappe de chaîne que le roseau d'écartement tient la plus éloignée de la tisseuse.

Pour les lisses, à strictement parler les boucles formées par la cordelette qui relie les fils pairs à la barre de lisse, une trentaine de notations sont disponibles. Les parlers chleuhs proposent inLitn, pluriel dont le singulier inLi a été retenu au Mzab, sans doute dans le Chenoua, et se trouve employé dans l'Aurès. La tamazight ne connaît, semblet-il, que asNli et son pluriel isNlitn qui serait peu employé; asNli est aussi attesté chez les Beni Snous et dans le Touat-Gourara. Les Kabyles disent ilni, pl. ilnan. Enfin, à inLi on paraît préférer dans l'Aurès l'appellation nuli. Toutes ces appellations ont en commun les consonnes $n$ et $l$ suivies au singulier d'une voyelle finale, l'hypothèse d'une racine commune est donc tentante.

[...] Pour inLi, pl. inLitn et asNli, pl. isNlitn, appellations qui nous valent les deux tiers des notations, elle est probablement justifiée. La sifflante présente dans asNli n'est qu'un élément formatif à valeur factitive. Le $i$ initial de inLi peut s'expliquer par harmonie vocalique. Enfin les pluriels de inLi et de asNli sont tous deux à suffixe - tn, ce qui étaie l'hypothèse d'une racine commune. Celle-ci ne se réduit pas à la bilitère NL. Le féminin 
tinLi « fil (en général)» est en effet largement attesté dont les pluriels démontrent l'existence d'une dernière radicale W. La racine commune postulée serait donc NLW. Aucune forme verbale correspondante n'est attestée pour renseigner sur son sens.

Le terme ilni, pl. ilnan - schèmes parfaitement berbères - ne désigne les lisses qu'en Kabylie et paraît seul pouvoir les y désigner. Il n'existe pas de verbe vivant auquel rattacher ce mot. Pour le rapporter à la même racine que les appellations précédentes, on doit invoquer une métathèse $-n l->-\ln$ - qui se trouve avoir été signalée: anli et alni « cervelle » coexistent par exemple chez les Ayt Youssi du Moyen-Atlas.

[...] Dans l'Aurès, où seulement elle s'emploie, domine l'appellation nuli. L'absence de voyelle initiale ne fait pas problème, c'est un trait dialectal conditionné par la structure du mot. Mais avec un u entre le $n$ et le $l$ ce terme ne semble pas devoir être rattaché à la racine NLW. Et s'il s'y rattachait, comment expliquer qu'il coexiste avec inLi pour évoquer dans un même parler le même signifié? En fait nuli paraît être un terme étendu du lexique chaouïa du métier horizontal à un rang de lisses au lexique du métier vertical.

\section{Roseaux d'écartement et d'enverjure (B. 1963 : 217-225)}

Au-dessus des lisses, pris entre les deux nappes de fil de chaîne se trouvent un ou plusieurs roseaux. Le roseau indispensable, dit d'écartement, est un organe propre au métier à un rang de lisses. C'est en l'élevant et en l'abaissant qu'on ouvre alternativement le pas et le pas inverse pour la duite, c'est-à-dire le passage du fil ou du brin de trame. Un, deux ou trois roseaux, rendus solidaires ou non, sont habituellement introduits au-dessus du roseau d'écartement, à proximité de l'ensouple supérieure. Ce sont les baguettes d'enverjure. Elles servent de point d'appui à un ou plusieurs croisements de la chaîne effectués lors de l'ourdissage en vue d'éviter le chevauchement des fils quand ils seront distribués sur toute la longueur de l'ensouple.

C'est pour ces roseaux, celui qui est indispensable comme celui ou ceux qui sont facultatifs, que la littérature sur le métier vertical nord-africain est la moins riche. Juste une vingtaine de notations, ne permettant généralement pas de distinguer entre les deux organes quand elles n'y assimilent pas en plus la barre de lisse, elle-même souvent faite d'un roseau. Le nom vernaculaire du roseau permet à lui seul la désignation technique. Sauf dans le Mzab, c'est de l'Atlantique à Siwa le berbère aġanim, pl. igunam ou iġanimn. Ce mot a été rapproché du pluriel punique qanim « roseaux ». Chez les Ayt Hadiddou, la baguette d'enverjure reçoit une appellation propre tout à fait particulière, šnšiLu, que l'on retrouve dans le Touat-Gourara sous la forme knkiLu. Bynon a émis l'hypothèse d'une origine latine à partir du mot cancellus « treillis, barreau, balustrade ».

\section{Le fil de chaîne (B. 1963 : 165-185)}

La chaîne tendue entre les ensouples est ourdie à partir d'un fil de laine fin et résistant. Pour fabriquer ce fil on a sélectionné les plus longues mèches des toisons lavées et séchées et l'on a peigné la laine pour aligner ses fibres. Puis en étirant progressivement la masse floconneuse ainsi obtenue en même temps qu'on fait rouler la laine entre le pouce et l'index et tourner un petit fuseau auquel se raccorde le fil, les fibres sont tordues assez serrées; le fil est alors bobiné sur le fuseau pour conserver sa torsion. Il existe plusieurs dénominations. [...] Hormis dans le domaine chleuh, la plus répandue se retrouve partout jusqu'à Siwa; 


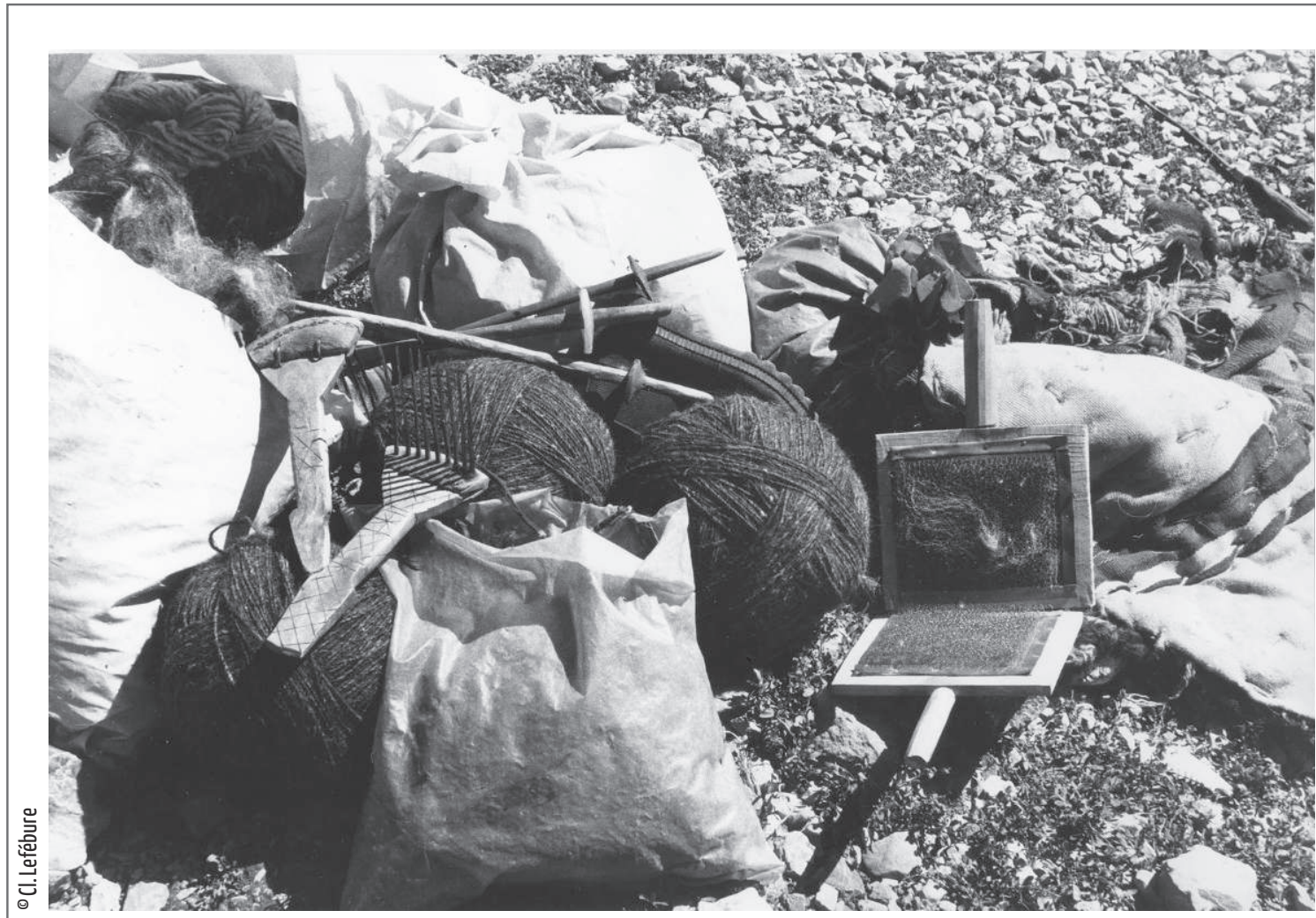

elle paraît être exclusive de toute autre dans de vastes régions de l'Algérie. C'est le masculin ustu, pl. ustan. Ni la racine ni le sens de ce mot n'invitent à le rapprocher du berbère $z d$ « tisser ». Mais on connaît la racine arabe classique SDW/SDY, à quoi se rattache par exemple sda en arabe marocain, et l'hébreu šeti i, ces deux appellations ayant le même sens qu'ustu/usti. Il est donc permis de penser à un emprunt du berbère au punique ou à un héritage du fonds chamito-sémitique.

[...] La dénomination la plus largement attestée après la précédente est idd. Elle semble être exclusive de toute autre dans le domaine chleuh, entre en compétition avec ustu en tamazight et se trouve signalée deux fois pour la Kabylie. Les enquêteurs n'indiquent jamais de pluriel pour ce terme, ce doit être un collectif. Galand (1970) a développé l'hypothèse d'une origine à partir de la racine BDD « se tenir debout ». L'appellation idd se justifierait par la verticalité du fil de chaîne quand il est tendu entre les ensouples.

[...] La troisième dénomination un tant soit peu attestée, asaraw, ne vaut, avec ce sens, que dans le Rif. Elle y entre en compétition avec ustu et comme ce terme prend parfois le sens de «frange ». Cela s'explique, la frange d'un tissage n'étant rien d'autre que les fils de chaîne retorsadés ou tressés qui dépassent aux extrémités. En Kabyle, asaru, terme peut-être parent, désigne « un cordon tressé ». Le féminin tasarut est bien connu avec le sens de «clé »; il proviendrait du verbe sri « dénouer » et aussi « peigner la laine » -soit « la faire s'ouvrir, la démêler». Avec asaraw la dénomination a pu vouloir prendre en compte que ce fil est filé à partir de laine peignée.

[...] Un dernier terme, igrrsan (n) uzTa, ne se trouvait avoir été signalé qu'une fois, chez les Ayt Seghrouchen du Moyen-Atlas, avec le sens de « fil de chaîne ». Mais cette acception 


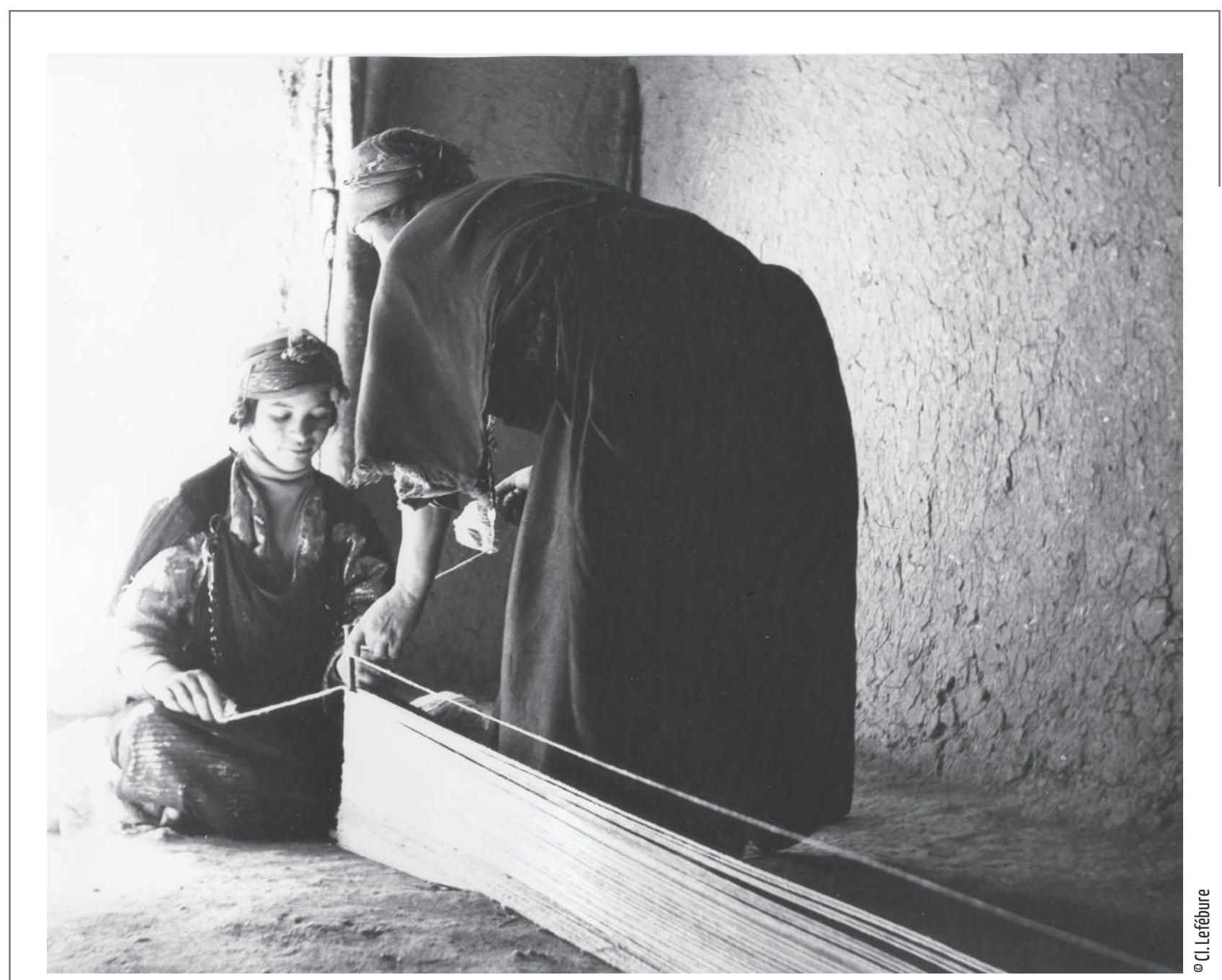

L'ourdissage de la chaîne

est à l'évidence celle de la forme ig risn que j'ai recueillie dans le parler des Ayt Atta. Ces deux pluriels sont bien attestés dans le vocabulaire du tissage. Cependant, le mot désigne habituellement les résidus de fil qui pendent de l'ensouple supérieure après que le tissage terminé en a été détaché en sectionnant la chaîne au moyen d'un couteau. Cette opération se décrit couramment en utilisant le verbe $B i$ « couper» mais le verbe $\dot{g} r s$ « égorger » peut aussi être employé. Fondamentalement, les igrrsan / ig risn ce sont les fils qui restent après « l'égorgement» du tissage ». Le mot s'est étendu à la désignation du fil qui sera « égorgé ». Dénomination lourde de sens, sur laquelle je reviendrai.

\section{Le fil de trame (B. 1963 : 186-204)}

Cordon continu ou suite de brins plus ou moins courts, les fibres que la tisseuse introduit à la main entre chaque croisement des deux nappes de chaîne ont été tordues moins serrées que le fil de chaîne. Cela donne, pour moins de travail, un fil moins résistant 
mais resté souple en épaisseur et qui s’avère propre à recouvrir complètement le champ du tissage après tassage au peigne; neuve, l'étoffe peut même présenter l'aspect du feutre. Cet élément de trame est filé à l'aide d'un gros fuseau à partir de laine courte cardée pour la nettoyer à fond et en aligner les fibres.

[...] Deux dénominations issues d'une même racine, celle du verbe $L m$ « filer », représentent les quatre cinquièmes de la cinquantaine de notations recueillies.

La plus répandue, tilmi, domine largement en tamazight et paraît exclusive en chleuh et en chaouïa. Le terme est un collectif et ne connaît pas de singulier. En chleuh, dans le Rif, chez les Beni Snous, tilmi signifie aussi « filage ». Comme dans le cas de azTa « action de tisser » mais aussi « ce que l'on tisse, ce qui a été tissé », il y a eu concrétisation du sens du nom d'action.

La seconde dénomination dérivée du verbe Lm, ulman, est usuelle dans les parlers berbères algériens moins le chaouia et a été notée sporadiquement en tamazight. Cette forme à initiale $u$ - paraît plus normale que la forme tilmi pour un verbe de la structure de $\mathrm{Lm}$. Ceci, joint à la distribution sporadique du terme là où domine tilmi, laisse penser que ulman est la dénomination la plus ancienne. La forme évoque un pluriel, à valeur de collectif. [...] L'appellation amŠim ne vaut que dans le Rif. Ce mot ou le féminin correspondant sont assez largement attestés avec des sens plus ou moins proches. En tamazight le signifié est une « trame grossière » comme on en file pour confectionner des sacs à grain, ou une « trame de laine et de poils de chèvre mêlés » servant à tisser les éléments de velum, ou encore une «mèche de laine cardée ». Dans les parlers du nord-ouest de l'Algérie, en kabyle, en chaouia, au Mzab, le signifié devient un «flocon de laine et/ou de neige ». Il est clair que l'appellation amŠim dénote l'aspect lâche du fil de trame.

[...] Un dernier groupe de notations minoritaires peut être rapidement considéré.

Chez les Beni Snous, l'emprunt berbérisé Nird vient de n-nîra, terme s'appliquant dans l'arabe du même groupe au fil de trame mais qui ailleurs désigne « les lisses ».

Les mêmes Beni Snous appellent encore aqrdaš le fil de trame. Ce nom désigne habituellement en berbère « une paire de cardes ». Cependant, avec qrda ̌ « cardes + fil de laine pour la trame + laine à brins courts pour la préparation de ce fil » l'arabe nordafricain témoigne de la même extension de sens.

Le kabyle connaît l'appellation adraf. Étymologiquement, le mot pourrait provenir de la berbérisation du pluriel traf de l'arabe nord-africain țrf « bout, morceau, fragment » dont la racine se retrouve dans le verbe $d R f$ « mettre de côté, écarter » connu en kabyle. La dénomination soulignerait la qualité seconde de la laine utilisée. Une autre étymologie serait métaphorique: adraf étant censé traverser la chaîne comme idrf « un sillon » traverse le champ, les deux mots ont le même pluriel idrfan. [...]

\section{Le peigne à tasser la trame (B. 1963 : 131-146)}

Après chaque duite, le fil ou le brin de trame est incorporé à la partie d'étoffe déjà tissée en le tassant énergiquement à coups d'un lourd peigne de fer [...]. Une cinquantaine de notations ont été relevées. Près des trois quarts correspondent à une dénomination unique quoiqu'attestée sous diverses formes, le reste d'une part à un terme propre au kabyle, d'autre part à deux emprunts à l'arabe.

[...] Les notations les plus nombreuses peuvent se ramener aux trois formes taz Ka, entendue au Maroc sauf dans le Rif, et dans le nord-ouest de l'Algérie; tadŠa, entendue dans le Rif, 


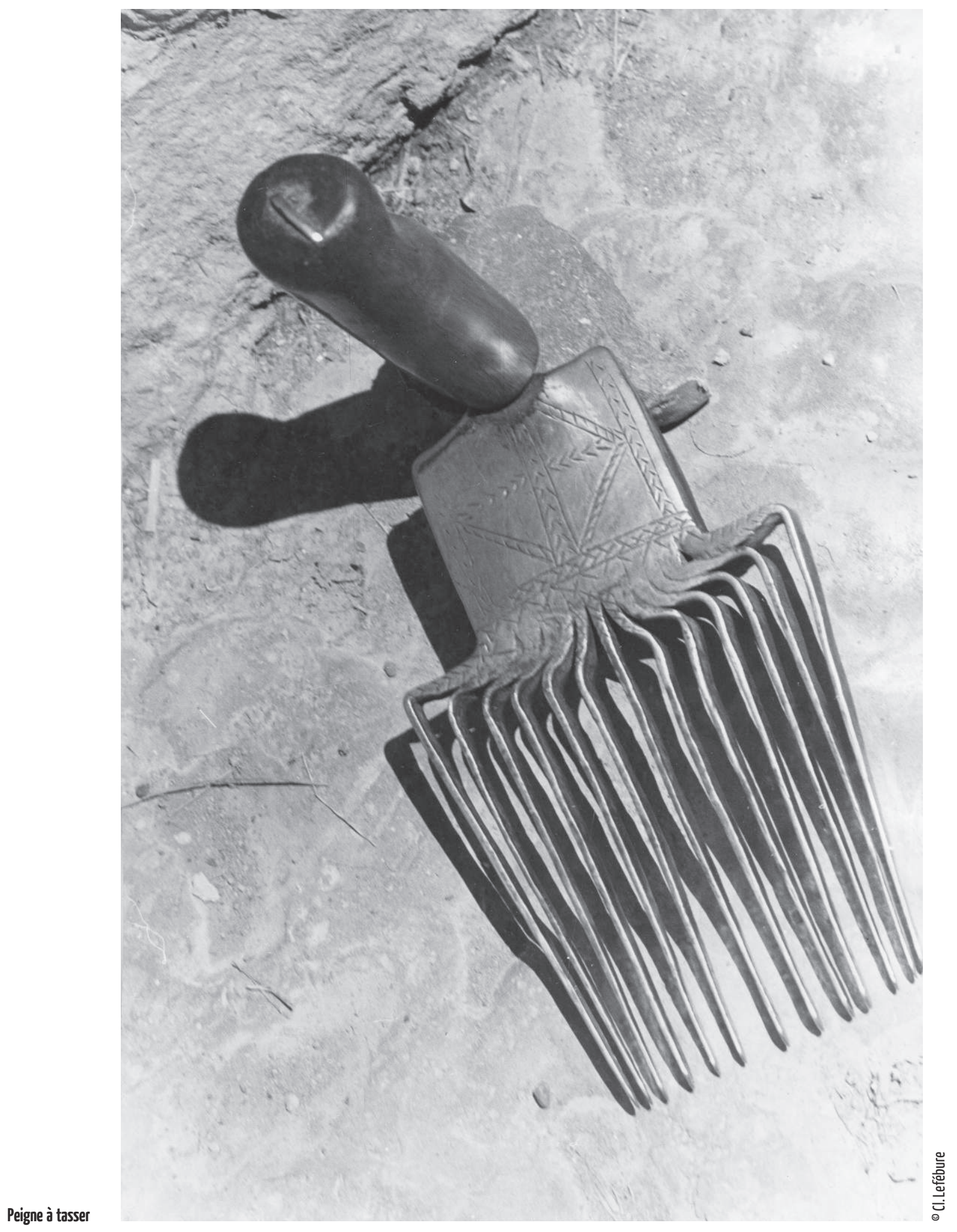


dans l'Aurès, et dans le sud-est algérien; taša, entendue dans le Mzab. Le rapprochement de ces formes serait osé si un mot de semblable structure ne venait témoigner d'une même diversité de réalisations. Ainsi « demain » se dit-il azKa/azka/asKa/aska dans tout le Maroc berbérophone moins le Rif, ad $\check{C} a$ dans l'Aurès, et $a \check{S} a$ au Mzab.

Quelle peut être l'étymologie de cette appellation? Une expression composée rifaine, employant pour le nom même du peigne l'un des emprunts arabes examinés infra, s'analyse en « peigne à $t z \check{S} a$ ». Notre terme semble donc être initialement un nom d'action; comme pour azṬa et tilmi le sens se serait ensuite concrétisé. Si l'on pense à « tassage », un verbe arabe y correspond: dekk « bourrer, tasser, damer ». Mais aussi, un verbe berbère: șk,șč « bâtir » qui a beaucoup reculé devant l'arabe bnu mais reste employé en Libye et en Mauritanie. En Afrique du Nord, « bâtir » c'est traditionnellement damer de la terre dans un moule, geste répétitif qui n'est pas sans rapport avec celui de la tisseuse quand elle tasse la trame.

[...] Dans toute la Kabylie, et seulement là, prévaut un autre nom noté sous les deux formes du masculin ayazill, pl. iyuzal et du féminin tayazilt, pl. tiyuzal, tiyazilin. Un auteur l'avait rapproché d'une « racine ZL qui a fourni le nom du fer dans tous les dialectes », ce qui ne tient pas compte du y pourtant probablement radical ni de l'emphase de la sifflante. Le problème étymologique attend sa solution.

[...] Quatre notations éparses ont pour origine la racine arabe HLL dont dérive le nom du peigne à tasser dans les parlers arabes d'Afrique du Nord autres que marocains. À Siwa, le peigne serait dénommé tumšit, terme qui provient de la racine arabe MŠḌ évoquant l'idée de « peigner ».

\section{Les tendeurs latéraux du tissu (B. 1963 : 122-130)}

Pour ne pas pocher en son milieu et afin que le dispositif des lisses puisse fonctionner bien, le tissu en cours d'élaboration doit être tendu le plus près possible de son bord supérieur. Cette tension est assurée au moyen de deux pinces, faites en fer ou en bois, prenant chacune sur une lisière du tissu et qu'une cordelette tire vers le plus proche montant. Une vingtaine de noms sont cette fois disponibles, partagés entre les idées de « tirer » et de « mordre » chacune évoqué par une racine berbère et une racine arabe. [...]

\section{Linguistique et technologie culturelle}

\section{Origine des termes, origine de la technique}

Pour désigner le métier dans son ensemble, nous avons vu qu'il n'existait de la côte atlantique aux marges orientales de la Libye qu'un seul et même terme berbère, de racine ZDW. Cette distribution d'ampleur à peu près maximale et le fait que l'érosion de la troisième radicale $\mathrm{a}$, au singulier comme dans le verbe, partout triomphé laissent croire que le terme date d'une période ancienne, antérieure au morcellement de la langue. Pour les ensouples aussi nous avons rencontré un terme unique, très largement attesté. Cette ample distribution et la constance de la forme plaident à nouveau en faveur de l'ancienneté d'un 
terme d'allure parfaitement berbère. Outre la désignation du métier dans son ensemble et celle des ensouples, seule celle des lisses s'effectue, sinon au moyen d'un terme unique peut-être au moyen de termes issus d'une même racine; encore n'est-ce pas l'hypothèse la plus probable, c'est plutôt la diversité du lexique qui s'affirme ici.

Celle-ci est en tous cas manifeste avec les quatre groupes de termes désignant les montants: deux d'entre eux sont secondaires, l'appellation de racine RGL est sans doute d'origine latine, le terme de racine NDW qui connaît la distribution la plus étendue est probablement d'origine berbère. Pour les roseaux tous confondus nous avons rencontré une appellation générique d'origine berbère ou punique, pour la baguette d'enverjure un mot d'origine peut-être latine. Deux noms du fil de chaîne se partagent la presque totalité du domaine: ustu est probablement d'origine punique, iD certainement d'origine berbère. Pour le fil de trame, les appellations principales sont fondées sur une racine berbère, le mot amŠim

l'est sans doute aussi. Pour le peigne à tasser, les trois termes principaux découlent d'une forme commune suffisamment ancienne pour avoir pu évoluer de manière ainsi diversifiée.

Au total nous aurions: d'origine punique, à moins qu'ils remontent au fonds commun chamito-sémitique, le nom principal du fil de chaîne et peut-être le nom générique du roseau; deux mots d'origine peut-être latine - l'un de distribution réduite, l'autre fort peu attesté; des apports arabes minoritaires; enfin une majorité d'étymologies berbères parmi lesquelles celles des seules appellations coextensives au domaine berbère du métier à tisser. Le vocabulaire du métier vertical en usage chez les berbérophones est fondamentalement indigène; rien n'invite à penser qu'il puisse en aller autrement de l'appareil lui-même. [...]

\section{Structuration lexicale et perception des techniques}

La spécialisation du lexique berbère du métier vertical n’a rien d'extrême. La racine de bien des termes n'évoque qu'une idée générale. Cependant nombre de ces idées s'opposent ou s'harmonisent comme s'opposent ou s'harmonisent matériellement les éléments du métier. [...] Le couple des mots $a z T a$ et tazKa, n'est pas sans évoquer les rapports complexes perceptibles entre ces éléments. Les fils de la chaîne comme les dents du peigne sont parallèles mais ont à se croiser perpendiculairement; la chaîne que l'on a tendue guide les dents du peigne qui tasse, mais celles-ci la transpercent; alors que les nappes de chaîne avancent et reculent alternativement très peu dans le plan horizontal, le peigne s'anime de haut en bas avec force et amplitude; le fer est rude en regard de la laine mais son poli vient d'elle. C'est toutes ces similarités et ces différences qu’on peut croire véhiculées par azTa et tažKa en vertu, d'une part de leur schème identique et de leur similarité phonique contrastant avec leur différence en genre grammatical, d'autre part du caractère distinct mais contiguë de leurs signifiés: « tissage » et « tassage » ne sont pas une même action mais « tissage, c'est tassage », la fatigue localisée des tisseuses en témoigne.

\section{Motivation linguistique et tendance technique}

Létude étymologique de idd m’a amené à citer l'article de L. Galand où il est montré, à propos des noms du fil de chaîne en berbère, en latin et en français, comment « la similitude des techniques entraîne parfois celle des procédés lexicaux » (1970 : 245). Je voudrais traduire cette leçon en termes de linguistique générale grâce à la notion de motivation et la mettre en 


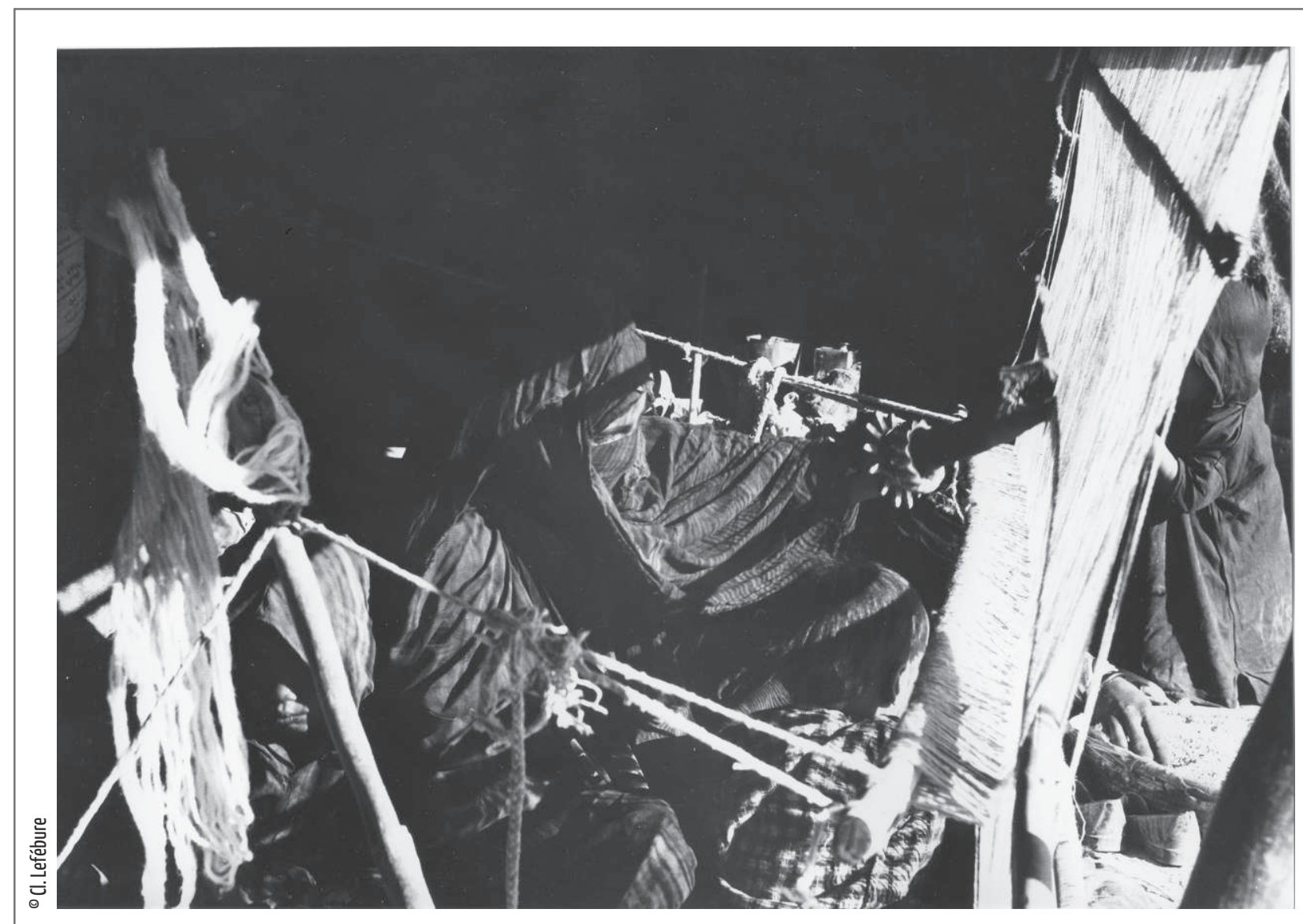

Tissage sous la tente

rapport avec la notion de tendance avancée par A. Leroi-Gourhan. Saussure situait l'arbitraire du signe dans la relation entre signifiant et signifié. Benveniste a fait du seul rapport du signe au référent le domaine de l'arbitraire, le lien entre le signifiant et le signifié est au contraire nécessaire. Il s'ensuit que le type d'évolution propre aux signes linguistiques, Saussure dit leur « mutabilité et immutabilité » (1968 : 108), caractérise le rapport du signe au monde, autrement dit « la motivation objective de la désignation, soumise à l'action de divers facteurs historiques » (Benveniste 1966 : 53). Leroi-Gourhan a fait plusieurs fois fructifier la notion de tendance énoncée dès le début de son premier ouvrage d'ethnologie des techniques, le plus simple est de reproduire les dernières lignes de sa réédition:

«Ce terme m'a paru, au début d'une recherche des lignes principales de la technicité humaine, nécessaire pour exprimer ce qui se situe en deçà de l'acte technique matérialisé dans les gestes et les instruments, terme de simple abréviation pour caractériser d'un mot la somme des virtualités qui ne deviennent réalités que dans les conditions de milieu favorable...» (1971: 326).

À cette « face abstraite du déterminisme évolutif », une et universelle, correspond et s'oppose sa « face concrète », les faits, qui incarnant la tendance dans un milieu technique donné, y acquièrent un cachet propre par degrés aux emboîtements historiques et sociologiques (1971 : 27-35; 1973 : 336-340). 
Larticle de Galand, outre qu'il présente l'analyse formelle de la dérivation de iD à partir de $b D$ « se tenir debout », l'étaye sémantiquement grâce à l'étymologie bien établie du nom du fil de chaîne en grec et en latin; en retour la polysémie de iD permet de donner son sens à l'évolution sémantique des vocables latin et vieux français. Le grec stêmôn et le latin stāmen se rattachent à la racine indo-européenne *sta- : ste-qui a donné gr. istêmi « mettre debout »et lat. stāre « se tenir debout». Ces dénominations sont pertinentes puisque Grecs et Romains tissaient sur des métiers verticaux. Mais le contraste fondamental du fil de chaîne et du fil de trame a valu à stāmen l'acception supplémentaire de « fil ayant les qualités requises de la chaîne » acheminant déjà vers le sens de « laine dont les fibres peuvent donner un fil ayant les qualités requises de la chaîne » qu'auront les représentants de stāmen en vieux français. Au xiII ${ }^{e}$ siècle, estain désigne une « laine peignée destinée à former la chaîne du drap » tandis qu'estame conserve les deux sens « fil de laine de première qualité » et « chaîne à tisser ». Au xviII siècle, estame n'est plus qu'une « laine peignée ». L'évolution sémantique qui a fait reculer l'idée de " chaîne » derrière celle de « laine d'une qualité particulière » a été favorisée par l'effacement des liens qui rattachaient estain et estame à la famille de stāre et par le remplacement du métier vertical par le métier horizontal à deux rangs de lisses.

Une chaîne à tisser ne saurait être habillée de trame sans avoir été tendue, c'est en cela que le tissage mérite d'être distingué de la vannerie (Leroi-Gourhan 1971 : 269). Mais si tendre une - métier à poids - ou deux nappes de fils peut se faire dans n'importe quel plan de l'espace, des avantages déterminants s'attachent à ce que cela ait été fait verticalement: d'une part la facilité de trouver une position de travail adaptée à la station et la gestualité humaines, d'autre part un encombrement minimal au sol d'où la facilité de mettre le tissage et celle qui tisse à l'abri des inconvénients du plein air - phénomènes climatiques, souillures, regards inopportuns, etc. On pourrait déceler une tendance du fil de chaîne à la verticalité. Quand d'entre tous ses caractères: tension, verticalité, solidité, continuité, etc., c'est de manière récurrente sa verticalité qui a été retenue pour le nommer, ne doit-on pas reconnaître que la motivation objective de la désignation s'accorde avec la tendance technique, qu'elle en est la traduction sur le plan de la langue? Les mêmes berbérophones qui appellent iD le fil de chaîne ont dénommé les fuseaux iz̧di et tiz̧dit ce qui renvoie à la racine berbère ZDY « être pesant » et traduit donc partiellement la tendance « poids-rotation-bobine » qu'incarne le fuseau (Leroi-Gourhan 1971 : 324) [...].

\section{Franges sémantiques, sens du tissage: l'apport des connotations}

[...] En gros, la dénotation c'est cette partie de la signification qui se caractérise par sa constance et sa valeur référentielle, tout ce qui s'y ajoute c'est la connotation.

\section{Tissage et créativité}

D'expression villageoise ou composée par des semi-professionnels, la littérature orale tient une grande place dans la vie sociale des groupes berbérophones. Au Maroc les troupes de poètes musiciens itinérants sont restées particulièrement actives. Dans un chant recueilli en 1967 (Harries 1970), le Maître d'un de ces groupes a un prologue que je traduis:

« Louange à Dieu. Sans Lui saurait-on dévider le poème.

Louange à Dieu. Sans Lui saurait-on n'en pas perdre le fil. 
Qu'alternent les mots! Qu'ils vous livrent mes réflexions.

Qu'alternent les mots! Leurs motifs sont à ma disposition.

La tisseuse et moi, les figures sont notre lot:

Nous activons-nous à l'une, c'est à la prochaine qu'il faut penser. »

La création poétique et le tissage - plus exactement le façonnage (Leroi-Gourhan 1971 : 282) - de motifs décoratifs sont comparés en vertu du fait qu'ils reprennent des modèles ce qui entraîne un partage de l'attention entre exécution et remémoration sélection. « Modèles » ne doit pas faire penser à quelque version de référence du poème ni à un carton de tapissier mais renvoie à une esthétique qui est moins d'originalité que de reprise. Ainsi le décor façonné combine-t-il des motifs de base dont l'inventaire est techniquement limité, ainsi la poésie traditionnelle, art d'à-propos et d'arrangement, doit-elle puiser à un fonds indivis: métier à tisser, métier à citer.

Les deux derniers vers de ce prologue développent une métaphore « chaîne à tisser $=$ poème $»$ suffisamment classique en poésie berbère ${ }^{9}$ pour que le premier distique l'ait convoquée par ellipse. Littéralement celui dit en effet:

« Louange à Dieu. Cet azTa-là il n'y en a pas sauf sous Sa main (bis)».

et tout le monde comprend. Par elle-même l'image n'implique pas spécialement le tissage à décor façonné. Son développement dans ce sens a cependant seul capté l'intérêt de J. Harries (1971), encore privilégie-t-elle la «trame », les vers en partie préconstruits du poème lui paraissant s'empiler comme les bandes décorées du tissage. Mais il existe une forme poétique berbère qui satisfait pleinement à l'analogie proposée: celle que son cadre grammatical structure à la fois verticalement et horizontalement et que P. Galand-Pernet (1969) a décrite pour cette raison sous le nom de «schème-grille».

Pour un esprit berbère, l'entrelacs des fils et celui des mots sont donc parents. Le nom de la chaîne à tisser et celui de l'ensouple, les noms du fil de trame et du motif décoratif, connotent à divers degrés l'idée de création littéraire. [...] Mais si la création littéraire est connotée, plus largement la créativité l'est aussi. Les associations sémantiques mises en évidence me paraissent à la fin signaler que le tissage berbère en est un terrain d'expression, domaine entr'ouvert à défaut de lui être voué à l'exercice de la liberté et de la responsabilité féminines. Dans le contexte maghrébin cela ne saurait être négligé.

\section{Tissage et dignité}

Une chanson kabyle enregistrée sur disque, « La quinzaine de chez Renault », exhorte l'ouvrier émigré à faire bon usage de son salaire. Sujet traditionnel; au deuxième couplet il est dit:

$$
\begin{gathered}
\text { «Qu'il évite l'ouvrier le chemin de l'ivresse } \\
\text { Qu'il suive celui de azTa } \\
\text { Ses ennemis entendront parler de lui ». }
\end{gathered}
$$

azTa compte ici pour « honneur, dignité » - Nif dans la culture. Le caractère rectiligne du métier lui vaut de pouvoir symboliser la première vertu morale de l'individu kabyle. La relation entre le Nif et ce qui est droit - et/ou à droite - a pu être établie dans une perspective structurale par P. Bourdieu (1972 : 36-37) [...]. Autre indice du lien entre honneur et métier à tisser en Kabylie, les serments les plus solennels se prêtent en jurant « par les nappes de la chaîne à tisser » ou « par cette chaîne que tissent les Anges » ou 


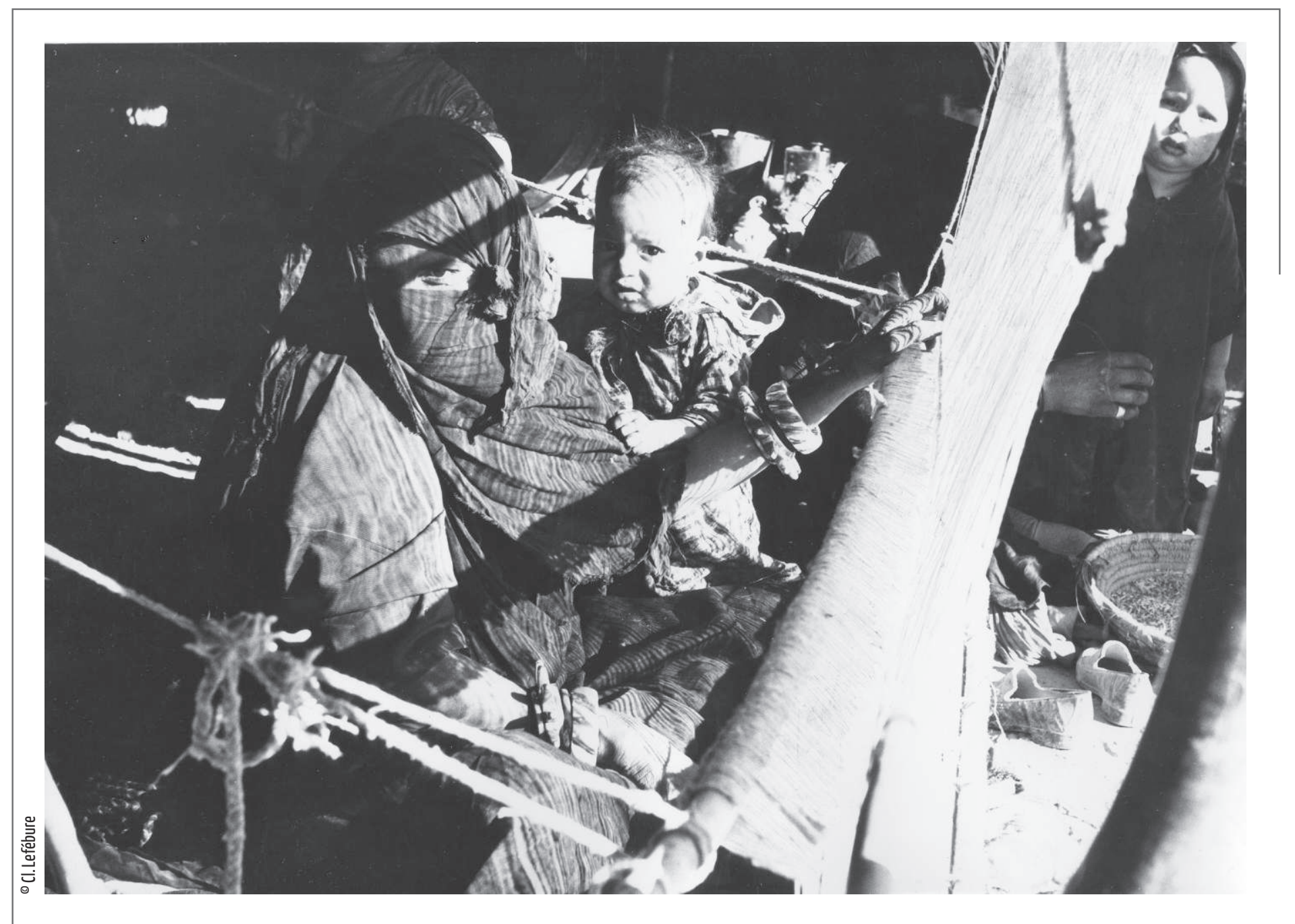

« Paravent de mon honneur »

« par cet enclos des Anges où il y a cent fils », etc. (Genevois 1967 : 25). Enfin le tissage garde à la maison les femmes, leur honneur pendant ce temps n'est pas en péril. Et grâce au peigne à tasser la plus pauvre peut éviter la déchéance. Les femmes concluent « Travail de la laine, paravent de mon honneur » (Genevois $1967: 7$ ).

Tissage et sacré

[Alors que les deux analyses qui viennent d'être conduites partaient d'une suggestion métaphorique c'est-à-dire d'un relais de sens, mon dernier développement naîtra de l'étymologie d'un des noms du fil de chaîne soit d'un infra-sens.]

Lappellation igrrsan, igrrisn « fil de chaîne » provient du verbe « égorger ». De fait, les cultures maghrébines prêtent vie et mort au tissage. C'est sur le terme ultime que l'appellation invite à se pencher, pour examiner un rituel qui reste respecté de nos jours [...]. La coupe se fait au couteau de boucherie ou avec des ciseaux. Ce sont habituellement les femmes qui l'effectuent, en Kabylie « de préférence une femme âgée et habile » (Genevois 
1967 : 71) parce que la femme âgée est moins femme. Mais à Tabelbala « c'est un homme qui doit le premier couper la chaîne (...). Il coupe une dizaine de fils d'un coup de son couteau rasoir. La formule consacrée pour demander cette aide est "viens égorger mon mouton" » (Champault 1969 : 236). Dans l'Atlas marocain (Boulifa 1908 : 198), chez les Beni Snous (Servier 1962 : 255), celle qui opère prononce la šahada, « le témoignage » (la ilaha iL uLah...), comme pour quelqu'un qui va mourir; à Bou Saada (Ricard 1928 : 221), en Kabylie (Genevois 1967 : 73), on invoque le nom de Dieu (bismiLah).

D'autres détails mériteraient d'être pris en compte. Ceux qui ont été relevés suffisent pour démontrer la dimension sacrée du dernier acte relatif au tissage et enrichir toute cette activité d'un sens qu'atteste une débauche d'autres rites. Rien ne légitime, et cette tentative au contraire condamne, la focalisation de l'attention de l'anthropologue sur le discours dénotatif. La description technique ne saurait beaucoup servir la technologie culturelle si elle devait se borner à l'enregistrement des chaînons opératoires les plus évidemment productifs. C'est la même option scientiste, à terme et par origine ethnocentrique, qui fait négliger dans le discours les connotations et dans les processus techniques leurs « pauses » symboliques. Sauf raisons majeures, l'indigène, lui, ne distingue pas. 
1. Les travaux considérables de A.-G. Haudricourt en la matière restent des études de terminologie historique et/ou comparée.

2. Dans la terminologie de Guiraud (1969 : 30) qui rappelle que certains auteurs préfèrent parler de sens et d'effets de sens, et d'autres de sens et de significations.

3. On se place ici au niveau de la langue et on s'y tiendra. Au niveau de la parole, l'expérience et l'affectivité de chacun peuvent ajouter à ce registre d'associations socialisées un supplément de sens purement idiosyncrasique dont la saisie est du ressort de la psychologie.

4. Même dans la culture occidentale, favorable à des termes techniques dont le sens contextuel et le sens de base se confondent - les noms scientifiques d'agents chimiques par exemple -, le discours technique n'échappe pas à la polysémie. Pensons par exemple aux mots « engrenage, courroie de transmission, détonateur, soupape de sécurité, etc. » que je choisis à dessein pour leurs champs sémantiques rattachant le monde du travail industriel à celui du discours révolutionnaire, ce qui ne saurait manquer de désigner une articulation pertinente.

5. L'arabe, aujourd'hui majoritaire, a rayonné à partir des villes et des steppes en direction des montagnes, à l'intérieur des plus massives desquelles il continue de le céder au berbère - langue non écrite, sauf exception, et morcelée en un très grand nombre de parlers; le français est également pratiqué. Bien qu'aucune enquête récente n'ait établi le nombre de gens dont le berbère est la première langue, on peut admettre qu'il avoisine présentement (2010) 22 millions. Les deux tiers de cet effectif se recrute au Maroc où les berbérophones représenteraient près de $40 \%$ de la population; l'Algérie compte 7 à 8 millions de ces locuteurs.
6. Sauf à Tabelbala où est parlé par exception un dialecte « au vocabulaire en majorité sonraï, mais riche de formes berbères et de racines arabes » (Champault 1969 : 43 - croquis et lexique du métier vertical p. 232).

7. Dépouillée par Bynon, la littérature fournit 84 sources pour le nom du métier à tisser vertical: 41 sont relatives aux dialectes marocains (11 pour le chleuh, 18 pour la tamazight, 12 pour le rifain), 31 sont relatives à l'Algérie (6 pour les parlers du Nord-Ouest, 16 pour le kabyle, 5 pour le chaouia de l'Aurès, 10 pour les parlers sahariens), 6 sont relatives aux parlers libyens et de Siwa. Quels que soient la partie du métier à tisser ou l'instrument qui seront considérés dans la suite de ce travail, nous disposerons de sources sensiblement aussi diverses sinon toujours aussi nombreuses (jamais moins d'une vingtaine).

8. La polysémie d'un mot comme azṬa n'a rien de spécifiquement berbère: Bynon cite le français tricot - nom d'action, de patient pendant l'action, de résultat de l'action - pour l'évident parallélisme de son champ sémantique (1963: 61).

9. J'en relève trois illustrations marocaines: en chleuh, un distique où le poète réclame « une ensouple - afGigafin d'ourdir la parole» (Justinard 1930 : 128); en tamazight, dans le prologue d'une cantilène inspirée de la légende biblique de Moïse (Laoust 1939 : 281): « Laissons ce chant - azTa-pour en entonner un autre Voici une nouvelle ensouple - afGag - et du fil de trame - ullum »; et dans le final d'une cantilène de Job (Laoust 1939 : 283): « Prions sur le Prophète. Dieu est maître de la récitation - azTa ». 


\section{RÉFÉRENCES}

Basset, A. 1952 La Langue berbère. London : Oxford University Press.

Bel, A. \& Ricard, P. 1913 Le Travail de la laine à Tlemcen. Alger : Jourdan.

Benveniste, E. 1966 Problèmes de linguistique générale. Paris: Gallimard.

Boulifa, S. 1908 Textes berbères en dialecte de l'Atlas marocain. Paris : Leroux.

Bourdieu, P. 1972 Esquisse d’une théorie de la pratique. Précédé de trois études d'ethnologie kabyle. Genève/ Paris : Droz.

Bynon, J. 1963 Recherches sur le vocabulaire du tissage en Afrique du Nord. Thèse pour le doctorat du III ${ }^{e}$ cycle. Paris.

Champault, D. 1969 Une Oasis du Sahara nord-occidental, Tabelbala. Paris : CNRS.

Cresswell, R. 1976 Les Bases d'un programme de travail. Techniques et Culture. Bulletin de l'Équipe de recherche 191, 1: 7-59.

Galand, L. 1970 Latin Stamen, français étaim, berbère idd "fil de chaîne". In Mélanges Marcel Cohen. Paris/ La Haye : Mouton : 245-253.

Galand-Pernet, P. 1969 Un "Schème-grille" de la poésie berbère: étude du motif des métamorphoses dans les poèmes chleuhs. Word XXV (1-3): 120-130.

Genevois, H. 1967 Sut-Tadut. La laine et le rituel des tisseuses. Fort-National, Fichier de documentation berbère.

Guiraud, P. 1969 La Sémantique. Paris : Presses Universitaires de France.

Harries, J. 1970 A Berber Tanshat: In a Tamazight Dialect. Bulletin of the School of Oriental and African Studies XXXIII (2) : 308-321.

Harries, J. \& Raamouch, M. 1971 Berber Popular Songs of The Middle Atlas. African Language Studies XII: 52-70.

Justinard, L. 1930 Les Ait Ba Amran. Paris : Champion.

Laoust, E. 1939 Cours de berbère marocain. Dialecte du Maroc central. $3^{e}$ éd. Paris : Geuthner.

Lapanne-Joinville, J. 1940 Les Métiers à tisser de Fès. Hespéris XXVII : 21-92.

Lemonnier, P. 1976 La Description des chaînes opératoires: contribution à l'analyse des systèmes techniques. Techniques et Culture. Bulletin de l'Équipe de recherche 191, 1: 100-151.

Leroi-Gourhan, A. 1971 L'Homme et la Matière. 2e éd. Paris : Albin Michel.

Leroi-Gourhan, A. 1973 Milieu et techniques. 2e éd. Paris : Albin Michel.

Ricard, P. 1928 Techniques et rites du travail de la laine en Algérie. In Mémorial Henri Basset. Paris : Geuthner : 207-227

Saussure, F. de. 1968 Cours de linguistique générale. Paris : Payot.

Servier, J. 1962 Les Portes de l’année. L’Algérie dans la tradition méditerranéenne. Paris : Laffont.

\section{NOTES ET RÉFÉRENCES DE L'INTRODUCTION}

Toutes les illustrations de l'article sont la propriété de l'auteur.

En ouverture, le signe typographique représenté est une esperluette - ligature de la conjonction et ; celle-ci fut conçue dans les années 70 par Claude Lefébure qui offrit ainsi un logotype à l'équipe de la revue Techniques \& culture dans sa belle genèse.

Lefébure, Cl. 1985 Réserves céréalières et société : l'ensilage chez les Marocains. In Gast, Sigaut, Beutler Les techniques de conservation des grains à long terme III. Paris : CNRS : 211-235. 